Draft Version November 8, 2018

Preprint typeset using $\mathrm{LAT}_{\mathrm{E}} \mathrm{X}$ style emulateapj v. 5/2/11

\title{
NON-ELASTIC PROCESSES IN ATOM - RYDBERG ATOM COLLISIONS: REVIEW OF STATE OF ART AND PROBLEMS
}

\author{
A.A.Mihajlov, V.A.SrećKović, LJ. M. Ignjatović \\ Institute of physics,Univesity of Belgrade, P.O. Box 57, 11001, Belgrade, Serbia
}

A. N. KLyucharev

Dept. of Physics, St-Petersburg University, Ulianovskaya 1, 198904 St. Petersburg, Petrodvorets, Russia

\section{M.S.DimitriJević}

Astronomical Observatory, Volgina 7, 11060 Belgrade 74, Serbia and Observatoire de Paris, 92195 Meudon Cedex, France and IHIS Techno experts, Batajnički put 23, 11080 Zemun, Serbia

AND

N.M. SAKAN

Institute of physics, Univesity of Belgrade, P.O. Box 57, 11001, Belgrade, Serbia

(Received June 2015; Revised revision date; Accepted acceptance date) Draft version November 8, 2018

\begin{abstract}
In our previous research, it has been demonstrated that such inelastic processes in atom Rydbergatom collisions, as chemi-ionization and (n-n') mixing, should be considered together. Here we will review the present state of the art and the actual problems will be discussed. In this context, we will consider the influence of the (n-n')-mixing during a symmetric atom Rydberg-atom collision processes on the intensity of chemi-ionization process. It will be taken into account $\mathrm{H}(1 \mathrm{~s})+\mathrm{H}^{*}(\mathrm{n})$ collisional systems, where the principal quantum number $n>>1$. It will be demonstrated that the inclusion of ( $\mathrm{n}-$ n') mixing in the calculation, influences significantly on the values of chemi-ionization rate coefficients, particularly in the lower part of the block of the Rydberg states. Different possible channels of the (n-n')-mixing influence on chemi-ionization rate coefficients will be demonstrated. The possibility of interpretation of the (n-n')-mixing influence will be considered on the basis of two existing methods for describing of the inelastic processes in symmetrical atom Rydberg-atom collisions.

Subject headings: atomic and molecular processes, plasmas, spectral line profiles
\end{abstract}

\section{INTRODUCTION}

Exploring and improving the new calculation possibilities and simulation techniques, attracted extensive attention in the chemi-ionization and (n-n')-mixing processes in atom Rydberg atom collisions, which resulted in numerous papers dedicated to this problem in various research fields like astrophysics, plasma physics, chemistry (see for example Bohr et al. (2012); Barklem (2007); Mihajlov et al. (2007a); Rvabtsev et al. (2005)).

Two groups of inelastic processes in slow atomRydberg atom collisions will be considered in this paper: the chemi-ionization processes,

$$
\begin{array}{r}
A^{*}(n, l)+A \rightarrow A+A^{+}+\vec{e} \\
A^{*}(n, l)+A \rightarrow A_{2}^{+}+\vec{e}
\end{array}
$$

and the processes of (n-n')-mixing

$$
A^{*}(n, l)+A \rightarrow A+A^{*}\left(n^{\prime}, l^{\prime}\right) .
$$

Here $A$ and $A^{*}(n, l)$ denote atom in the ground and in highly excited (Rydberg) state with the given principal and orbital quantum numbers $n$ and $l, A^{+}$and $\vec{e}$ - atomic

vlada,mihajlov@ipb.ac.rs

anklucharev@gmail.com

mdimitrijevic@aob.rs

nsakan@ipb.ac.rs ion in the ground state and free electron, while $A_{2}^{+}$denotes the molecular ion in the ground state.

The processes (1) and (2), illustrated by Figs. 1] and $1 \mathrm{~b}$, were examined and discussed in the literature for a long time (see e.g. Mihajlov \& Janev (1981); Janev et al. (1987)). These processes are conditioned by the dipole resonant mechanism which was described in details in Mihajlov et al. (2012). Significant contribution of processes (1) and (2) in modeling of solar atmosphere is shown in Mihajlov et al. (2011a b); Barklem (2007); Mashonkina (2009, 2010), while the papers of Mihajlov et al. (2003) and Srećković et al. (2013), are devoted to the influence of these processes on the kinetic of helium-rich star atmospheres. Another important thing is that the presented results suggest that these processes, due to their influence on free electron density and excited state populations in the atmospheres of $\mathrm{M}$ red dwarfs, should also influence the atomic spectral line shapes (see e.g. Mihajlov et al. (2007b)).

In spite of the fact that the processes (1) and (2) are caused by the same mechanism, they are considered separately up to now. The main aim of this work is to determine the influence of processes (2) on the processes of chemi-ionization (1a) and (1b). Namely, already from Fig. 1b one can notice the following: in the case when the considered atomic collision proceed in accordance with the excited molecular term $U_{2}(R)$, before it enters in the 


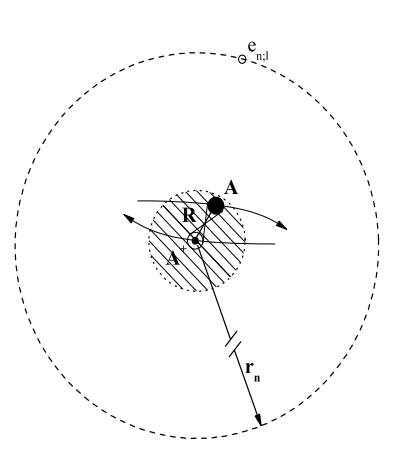

(a)

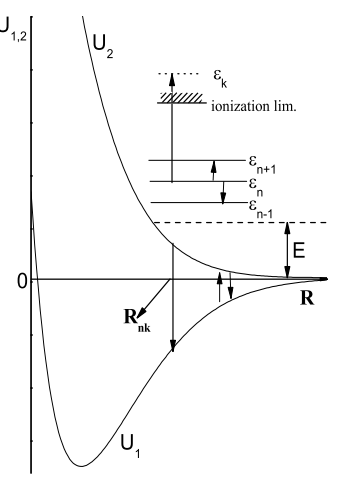

(b)

Figure 1. (a) Schematic illustration of $A^{*}(n, l)+A$ collision within the domain of internuclear distances $R \ll r_{n ; l}$, where $r_{n ; l} \sim n^{2}$ is the characteristic radius of Rydberg atom $A^{*}(n, l)$; (b) Schematic illustration of the simultaneous resonant transitions of the outer electron from the initial bound to the final state and the sub-system $A^{+}+A$ from initial excited to the final ground electronic state. If the outer electron becomes free $\varepsilon_{k}>0$ the processes (1) occur, while if the outer electron remains in the bound state $\varepsilon_{n^{\prime}}<0$ the processes 2 occur.

zone where the chemi-ionization processes (1a,b) occur, the system $A^{*}(n, l)+A$ passes through the zone where the processes (2) with $n^{\prime}>n$ take place.

As first, the way of inclusion of process (2) in the procedure of calculation of rate coefficients of the chemiionization processes $(1 \mathrm{a}, \mathrm{b})$ will be described. For this purpose their values will be determined under the conditions characteristic for the Solar photosphere in the case $A=\mathrm{H}(1 s)$ and compared with the rate coefficients of the same chemi-ionization processes, determined in Mihajlov et al. (2011a), but without inclusion of (n-n')mixing processes. We draw attention that, as a difference from this previous article, chemi-ionization rate coefficients are here without the simplification of the expression for Gaunt factor, connected with the photoionization cross sections for the transitions of Rydberg electron $\varepsilon(n, l) \rightarrow \varepsilon(k)$. Besides, here, as a difference from Mihajlov et al. (2011a), the average chemiionization rate coefficient for a given $n$ is obtained as a result of the corresponding averaging of partial chemiionization rate coefficients for every $l$ where $0 \leq l \leq n-1$.

Atomic units will be used throughout the paper.

\section{THE THEORY}

\subsection{General formulas}

Let $K_{1 a}(n, l ; T)$ and $K_{1 b}(n, l ; T)$ are rate coefficients of processes (1a) and (1b), separately determined for given $n, l$ and $T$ where $T$ is temperature of the considered plasma, and $K_{1}(n, l ; T)$ is the total rate coefficient of processes (1a) and (1b) together, namely $K_{1}(n, l ; T)=$ $K_{1 a}(n, l ; T)+K_{1 b}(n, l ; T)$.

Because of further applications, we will then determine the average total rate coefficient

$$
K_{1 ; n}(T)=\frac{1}{n^{2}} \cdot \sum_{l=0}^{n-1}(2 l+1) \cdot K_{1}(n, l ; T),
$$

and average rate coefficient of associative ionization

$K_{1 b ; n}(T)$

$$
K_{1 b ; n}(T)=\frac{1}{n^{2}} \cdot \sum_{l=0}^{n-1}(2 l+1) \cdot K_{1 b}(n, l ; T) .
$$

Partial rate coefficients $K_{1}(n, l ; T)$ and $K_{1 b}(n, l ; T)$ are determined on the basis of standard expressions

$$
\begin{gathered}
K_{1}(n, l ; T)=\int_{E_{n ; i}}^{\infty} \sigma_{1}(n, l ; E)\left(\frac{2 E}{\mu_{r e d}}\right)^{1 / 2} f_{T}(E) d E, \\
K_{1 b}(n, l ; T)=\int_{E_{n ; i}}^{\infty} \sigma_{1 b}(n, l ; E)\left(\frac{2 E}{\mu_{\text {red }}}\right)^{1 / 2} f_{T}(E) d E
\end{gathered}
$$

where $E$ is impact energy, $\sigma_{1}(n, l ; E)$ and $\sigma_{1 b}(n, l ; E)$ are the corresponding cross sections, $\mu_{\text {red }}$ is reduced mass of the subsystem $\mathrm{H}(1 \mathrm{~s})+\mathrm{H}^{+}$, and $f_{T}(E)$ is the Maxwell distribution function: $f_{T}(E)=\exp (-E / k T) \sqrt{E}$. Parameter $E_{n ; i}$ is given here with the relation $E_{n ; i}=U_{2}\left(R_{n ; i}\right)$ where $R_{n ; i}$ is the upper limit of the chemi-ionization zone which is the root of the equation $U_{12}=1 / 2 n^{2}$.

The mentioned cross sections are determined here within the semi-classical approximation, with the help of also standard expressions

$$
\begin{aligned}
\sigma_{1}(n, l ; E) & =2 \pi \int_{0}^{\rho_{1 ; \max }} P_{1}(n, l ; \rho ; E) \rho d \rho \\
\sigma_{1 b}(n, l ; E) & =2 \pi \int_{0}^{\rho_{1 b ; \max }} P_{1 b}(n, l ; \rho ; E) \rho d \rho
\end{aligned}
$$

where $\rho$ is impact parameter, $\rho_{1 ; \max }$ and $\rho_{1 b ; \max }$ - are the corresponding maximal values of this parameter, and $P_{1}(n, l ; \rho ; E)$ and $P_{1 b}(n, l ; \rho ; E)$ - are the total probability of chemi-ionization and the probability of associative ionization, respectively determined for the given values of $n, l, \rho$ and $E$. These probabilities we will determine here in the form

$$
\begin{gathered}
P_{1}(n, l ; \rho ; E)=\frac{1}{2} \cdot p_{\text {keep }}(n, l ; \rho ; E) \cdot p_{i ; 1}(n, l ; \rho ; E), \\
P_{1 b}(n, l ; \rho ; E)=\frac{1}{2} \cdot p_{\text {keep }}(n, l ; \rho ; E) \cdot p_{i ; 1 b}(n, l ; \rho ; E)
\end{gathered}
$$

where $1 / 2$ is the probability that the subsystem $\mathrm{H}(1 \mathrm{~s})+\mathrm{H}^{+}$develops in accordance with the term $U_{2}(R)$, $p_{\text {keep }}(n, l ; \rho ; E)$ - probability that in the domain of values of $R$ where the processes (2) with $n^{\prime}>n$ are possible, the state of this subsystem is held on, i.e. the excited electronic state with the energy $U_{2}(R)$, while $p_{i ; 1}(n, l ; \rho ; E)$ and $p_{i ; 1 b}(n, l ; \rho ; E)$ are the corresponding ionization probabilities determined under the condition that subsystem $\mathrm{H}(1 \mathrm{~s})+\mathrm{H}^{+}$enters in the ionization zone with probability equal to 1 .

\subsection{Probability of ionization decay}

Similarly as in the previous papers, probabilities $p_{i ; 1}(n, l ; \rho ; E)$ and $p_{i ; 1 b}(n, l ; \rho ; E)$ are determined here within the quasi-static decay approximation. Since these probabilities are determined in the similar way as in previous works of Mihajlov et al. (2007a); Mihajlov et al. (2011a), here they are taken in the form 


$$
\begin{array}{r}
p_{i ; 1}(n, l ; \rho ; E)=1.0-\exp \left(-2 q_{i ; 1}\right), \\
p_{i ; 1 b}(n, l ; \rho ; E)=\exp \left(-q_{i ; 2}\right) \cdot\left[1.0-\exp \left(-2 q_{i ; a s}\right)\right],
\end{array}
$$

where the quantities $q_{i ; 1}, q_{i ; 2}$ and $q_{i ; a s}$ are given as

$$
\begin{array}{r}
q_{i ; a s}=q_{i ; 1}-q_{i ; 2}, \quad q_{i ; 1}=\int_{R_{0}}^{R_{n ; i}} \frac{W_{i}(n, l ; R)}{v_{\text {rad }}(E, \rho ; R)} d R \\
q_{i ; 2}=\int_{R_{1 b ; \max }}^{R_{n ; i}} \frac{W_{i}(n, l ; R)}{v_{\text {rad }}(E, \rho ; R)} d R
\end{array}
$$

The rate coefficient of ionization decay $W_{i}(n, l ; R)$ and radial ion-atom velocity $v_{\text {rad }}(E, \rho ; R)$ are given by expressions

$$
\begin{gathered}
W_{i}(n, l ; R)=\frac{1}{2 \pi} \cdot c \cdot U_{12}^{3}(R) \cdot D_{12}^{2}(R) \cdot \sigma_{p h . i}\left(n, l, \varepsilon_{p h}\right) \\
v_{\text {rad }}(E, \rho ; R)=\left(\frac{2}{\mu_{\text {red }}}\left[E-U_{2}(R)-\frac{E \rho^{2}}{R^{2}}\right]\right)^{1 / 2}
\end{gathered}
$$

where $c$ is the speed of light, $D_{12}=|<1| \hat{d}_{m . i}|2>|$ - molecular-ion dipole matrix element, $\sigma_{p h . i}\left(n, l, \varepsilon_{p h}\right)$ cross section for photoionization of excited hydrogen atom $\mathrm{H}^{*}(n, l)$ by a photon with energy $\varepsilon_{p h}=U_{12}(R)$, and $U_{12}(R)=U_{2}(R)-U_{1}(R)$.

In the expression for dipole matrix element $\hat{d}$ denotes operator of ion dipole momentum $\mathrm{H}_{2}^{+}$and $\mid 1>$ and $\mid 2>$ - ground and first excited state of this ion.

In Eq. (11) with $R_{0}$ is denoted the lower limit of the domain $R$ which is reached during the collision with a given $\rho$ and $E$, and with $R_{1 b ; m_{i n}}$ the upper limit of the domain $R$ where is possible only the process of associative ionization (1b). Consequently, parameters $R_{0}$ represent here the roots of the equation: $U_{2}(R)=E \cdot\left(1-\rho^{2} / R^{2}\right)$ , and $R_{1 b ; \max }$-root of the equation: $U_{12}(R)=E$. Let draw attention that it is assumed in expressions (10) and (11) that $R_{1 b ; \max }<R_{n ; i}$ In the case of $R_{1 b ; \max }>R_{n ; i}$ we have that the quantity $q_{i ; 2}=0$, and $q_{i ; a s}=q_{i ; 1}$.

We draw attention that already at this point exist a difference compared to previous works concerning the chemi-ionization processes in stellar atmospheres (Mihajlov et al. 2007a; Mihajlov et al. 2011a). Namely, in just mentioned works, the chemi-ionization rate coefficients were determined with the averaged ionization decay rate, obtained by averaging of partial rates over the whole shell with given $n$. This gives possibility to use the average over shell Kramers photo-ionization crosssection adjusted with the help of approximate Gaunt factor. As a difference, the rate coefficients $K_{1}(n, l ; T)$ and $K_{1 b}(n, l ; T)$ were determined here on the basis of Eqs. (5) and (6) with the help of partial cross sections for photo-ionization, determined here on the basis of exact expressions from Sobelman (1979).

\subsection{Probability of pre-ionization decay}

From Eqs. (8) and (9) one can notice that the basic difference, in comparison with previous papers, represents direct taking into account of the effect of decay of the initial electronic state of the considered atom-Rydberg atom system, due to the possibility of execution of excitation processes (2) with $n^{\prime}>n$. This one takes into account by the introduction of probability of maintenance of this state $p_{k e e p}(n, l ; \rho ; E)$. One determines this probability on the basis of the modified version of approximate method described in Mihajlov et al. (2004) dedicated to the $\left(n-n^{\prime}\right)$-mixing processes. Let remind, that the essence of this method is that at given $n$ each block of Rydberg states from $n^{\prime}=n+p_{1}$ to $n^{\prime}=n+p_{2}$ is "spreading" in a part of "quasicontinuum" limited by values $n+p_{1}-\delta_{n}$ and $n+p_{2}+1-\delta_{n}$, where the parameters $\delta_{n}$ are determined from the condition of maintainance of total number of states and total oscillator strengths for transitions from initial state of Rydberg electron to all states of the separated block. The mentioned modification has been conditioned with the fact that in the just mentioned work was determined an average rate of decay of the initial state of system connected with the transition of Rydberg electron from the state with the given $n$ in states with $n^{\prime}=n+p$, where $p \geq 1$, while here we must to consider transitions of Rydberg electron from the state $\mid n, l>$ to the states $|n+p, l-1\rangle$ and $\mid n+p, l+1>$. In accordance with the just said, it is considered here that the preionization zone form the domain of internuclear distances such that $R_{n ; i}<R<$ $R_{n ; n+1-\delta_{n}}$, where $\delta_{n}=0.5 \cdot[1-(1 / 3) \cdot O(1 / n)]$, and domains $R$ corresponding to the mentioned transitions with $p=1,2,3 \ldots$ make intervals $\left(R_{n ; n+2-\delta_{n}}, R_{n ; n+1-\delta_{n}}\right)$, $\left(R_{n ; n+3-\delta_{n}}, R_{n ; n+2-\delta_{n}}\right),\left(R_{n ; n+4-\delta_{n}}, R_{n ; n+3-\delta_{n}}\right)$. The limits of these domains $R_{n ; n+p-\delta_{n}}$ are roots of the equations: $U_{12}(R)=0.5 \cdot\left[1 / n^{2}-1 /\left(n+p-\delta_{n}\right)^{2}\right]$.

In this work are taken into account the transitions with $1 \leq p \leq 5$. Consequently, the probability $p_{k e e p}(n, l ; \rho ; E)$ could be represented as

$$
p_{\text {keep }}(n, l ; \rho ; E)=\prod_{p=1}^{5} p_{p ; k e e p}(n, l ; \rho ; E),
$$

where $p_{p ; k e e p}(n, l ; \rho ; E)$ is the probability of the maintenance of the initial state of the system within the interval $\left(R_{n ; n+p+1-\delta_{n}}, R_{n ; n+p-\delta_{n}}\right)$.

Since the mechanism of the pre-ionization decay is the same as in the case of the ionization one, we take immediately that probabilities $p_{p ; k e e p}(n, l ; \rho ; E)$ are given with the relations

$$
\begin{array}{r}
p_{p ; \text { keep }}(n, l ; \rho ; E)=\exp \left(-x_{p}\right), \\
x_{p}=\int_{R_{n ; n+p+1-\delta_{n}}}^{R_{p}} \frac{w_{n ; n+p}(n, l: R)}{v_{\text {rad }}(E, \rho, R)},
\end{array}
$$

where the decay rate $w_{n ; n+p}(n, l ; R)$ is conditioned by the dipole mechanism within the interval $\left(R_{n ; n+p+1-\delta_{n}}, R_{n ; n+p-\delta_{n}}\right)$.

The upper limit $R_{p}$ is given by

$R_{p}=\left\{\begin{array}{l}R_{n ; n+p-\delta_{n}}, R_{n ; n+p-\delta_{n}} \leq R_{u p ; m i x}(E, \rho) \\ R_{u p ; m i x}(E, \rho), R_{n ; n+p-\delta_{n}}>R_{u p ; m i x}(E, \rho) \geq R_{n ; n+p}\end{array}\right.$

where $R_{n ; n+p}$ is the resonant distance of the process (2) for given $n$ i $n^{\prime}=n+p$, determined as a root of the equation

$$
U_{12}(R)=\frac{1}{2} \cdot\left[\frac{1}{n^{2}}-\frac{1}{(n+p)^{2}}\right]
$$


The parameter $R_{u p ; m i x}(E, \rho)$ is separately discussed in Appendix 1. Lets draw attention that here in the case when $R_{u p ; \operatorname{mix}}(E, \rho)<R_{n ; n+p}$ is considered that $p_{p ; \text { keep }}(n, l ; \rho ; E)=0$. In accordance with the above said, the decay rate $w_{n ; n+p}(n, l ; R)$ is given here by the relation

$$
\begin{array}{r}
w_{n ; n+p}(n, l ; R)=\frac{2 \pi}{3} \cdot U_{12}^{4}\left(R_{n ; n+p}\right) \cdot \widetilde{n}^{3} \cdot D_{12}^{2} \cdot r_{n, l ; n+p}^{2} \\
\tilde{n}=n \cdot\left[1-2 n^{2} \cdot U_{12}(R)\right]^{-1 / 2}
\end{array}
$$

where $r_{n, l ; n+p}^{2}=|<n, l| \hat{d}_{a t}|n, l-1>|^{2}+\mid<$ $n, l\left|\hat{d}_{a t}\right| n, l+1>\left.\right|^{2}, \hat{d}_{a t}$ - is the operator of dipole moment of hydrogen atom, and $|n, l>| n,, l-1>$ and $\mid n, l+1>$ denote the corresponding states of Rydberg electron.

\section{RESULTS AND DISCUSSION}

It follows from the above presented material that the total rate coefficients of the processes (1a) and (1b) together, and rate coefficients for the associative ionization (1b), i.e. $K_{1 ; n}(T)$ and $K_{1 b ; n}(T)$ are determined on the basis of Eqs. (3)-(17). Lets draw attention that, strictly speaking, chemi-ionization processes (1a) and (1b) can be described on the basis of dipole resonant mechanism only in the case of the state with $n \geq 5$, for which the potential curves of the system $\mathrm{H}^{*}(n, l)+\mathrm{H}(1 s)$ lay above the potential curve of the system $\mathrm{H}^{+}+\mathrm{H}^{-}\left(1 s^{2}\right)$, where $\mathrm{H}^{-}\left(1 s^{2}\right)$ is stable negative hydrogen ion. However, it can be shown that the points of the intersection of potential curves of the system $\mathrm{H}^{*}(n, l)+\mathrm{H}(1 s)$ with $n=2,3$ and 4 with the potential curve of the system $\mathrm{H}^{+}+\mathrm{H}^{-}\left(1 s^{2}\right)$ are located on the internuclear distances, which are several times larger than the average atomic radius $\mathrm{H}^{*}(n, l)$ so that the existence of these intersections can not significantly affect the values of the corresponding rate coefficients of the processes (1a) and (1b). Consequently the applicability of the dipole resonance mechanism for the states with $n<5$ depends to what degree may be regarded as fulfilled condition $R_{n ; n+1} \ll r_{n ; l}$ where $r_{n ; l}$ is the mean radius of the corresponding orbit of the outer electron. One notices that from this aspect, the dipole resonant mechanism can not be applied in the case of $n=2$, while in the case of the states $n=3$ and 4 the application of this mechanism can be completely justified.

Total values of the rate coefficients of chemi-ionization processes $K_{1 ; n}(T)$ within the range $3 \leq n \leq 15$ are presented in Tab. 11. Bearing in mind the main application, of here obtained results, on the photosphere and lower chromosphere of the Sun, calculations of these rate coefficients were performed here for temperatures $4000 \mathrm{~K} \leq T \leq 10000 \mathrm{~K}$. The processes $(1 \mathrm{~b})$ are characterized in this paper via the corresponding branch coefficient $X_{1 b ; n}(T)$ given as

$$
X_{1 b ; n}(T)=\frac{K_{1 b ; n}}{K_{1 ; n}} .
$$

Values of coefficients $X_{1 b ; n}(T)$ for the same $n$ and $T$ are presented in Tab. 2. In accordance with the above said, rate coefficients are determined here by summing the probability of the decay of the initial state of the collisional system in preionization zone with Rydberg elec-

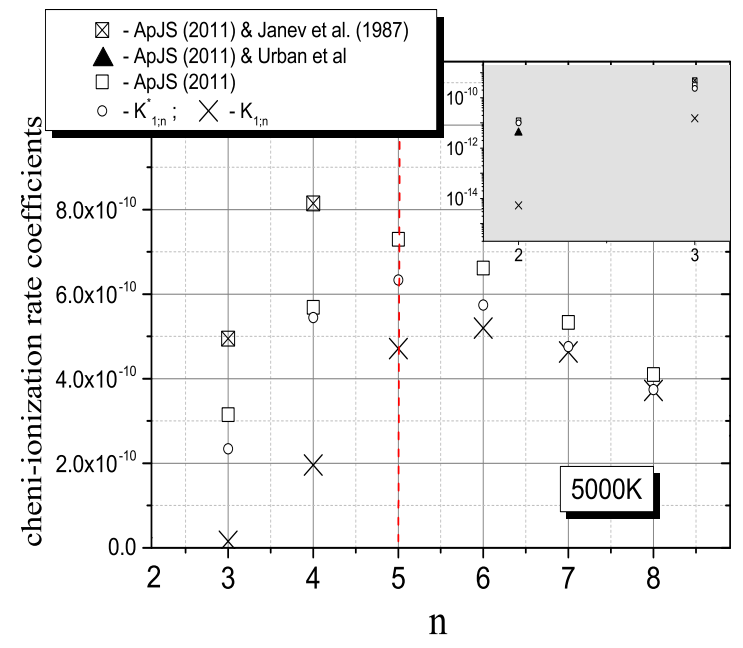

Figure 2. Comparison of the calculated values of rate coefficients of the chemi-ionization processes (1a) and (1b) with the data from Mihajlov et al. (2011a).

tron transitions from state $\mid n>$ to state $\mid n+p>$, where $1 \leq p \leq 5$.

In order to demonstrate significance of the presented calculation we will compare the chemi-ionization rate coefficients $K_{i ; n}(T)$ with the corresponding rate coefficients $K_{i ; n}^{*}(T)$ from Mihajlov et al. (2011a). Let us note that the coefficients $K_{i ; n}^{*}(T)$ are obtained in the same way as the coefficients $K_{i ; n}(T)$ but taking $p_{k e e p}(n, l ; \rho ; E)=$ 0 , where $p_{\text {keep }}(n, l ; \rho ; E)$ is the total probability of the preionization decay given by Eqs. (13) - (17). All mentioned quantities are presented in Fig 2 for the case of $T=5000 \mathrm{~K}$. Lets draw attention that in relation to the previous work of Mihajlov et al. (2011a) in this figure are presented not only the total rate coefficients, determined on the basis of dipole resonance mechanism for $3 \leq n \leq 8$ but also and rate coefficients determined there on the basis of data from Janev et al. (1987) for $n=3$ and 4, and from Urbain et al. (1991) for $n=2$. One can notice from this figure that there are noticeable differences between the values of the rate coefficients determined in Mihajlov et al. (2011a) and values $K_{i ; n}^{*}(T)$, while the differences in relation to the rate coefficients $K_{i ; n}(T)$ are very large for $n \leq 6$ and decrease quickly with the increase of $n$ in the area $n>6$.

In previous works (Mihajlov et al. 2003, 2007b) related to the photosphere of a $\mathrm{M}$ red dwarf with temperature near to $4000 \mathrm{~K}$, it has been shown that on populations of hydrogenic Rydberg states in this photosphere as well as on its other characteristics, influence strongly just the chemi-ionization processes (1a) and (1b) with $4 \leq n \leq 8$. It is clear that, already because of this, it is indispensable to take into account the changes of rate coefficients of these processes, which, in accordance with our results, are particularly large for $n \leq 6$. From the material presented here, follows also the great importance of the further investigation of the properties of decay of the initial state of the collisional system $\mathrm{H}^{*}(n, l)+\mathrm{H}(1 s)$ in the preionization zone.

Additionally, obtained here results suggest that the rate coefficients of the chemi-ionization processes (1a) and $(1 \mathrm{~b})$ could be affected and by other channels of in- 
fluence of the processes (2). Here we have in view the processes of (n-n') mixing taking place in two or more steps.

\section{CONCLUSIONS}

In the presented work is shown that the processes of (n-n')-mixing (2) influence considerably on the rates of chemi-ionization processes (1a) and (1b). Calculations, which characterize this influence on the quantitative level have been performed. As one can see from figure 2, inclusion of the (n-n') mixing processes into consideration, reduce the chemi-ionization rate coefficients. The obtained results are finalized in the tabular form, where the values of total constants for rates of the processes (1a) and (1b) together, and rates for the process of associative ionization $(1 \mathrm{~b})$ are presented. The tables cover the range of values, of the principal quantum number of Rydberg states of Hydrogen atom, from $n=3$ to $n=15$ and the temperature range from $T=4000 \mathrm{~K}$ to $T=10000 \mathrm{~K}$, so that they can be directly applied in connection with the modeling of photosphere and lower chromosphere of the Sun. Moreover, in the work have been discussed further directions of the investigation of the influence of (n-n')mixing processes on the chemi-ionization processes taking into account (n-n') mixing processes which occur in two or more steps.

The authors are thankful to the Ministry of Education, Science and Technological Development of the Republic of Serbia for the support of this work within the projects
176002, III44002 and 171014.

\section{REFERENCES}

Barklem, P. S. 2007, A\&A, 466, 327

Bates, D. R., \& Boyd, A. H. 1962, Proceedings of the Physical Society, 80, 1301

Bohr, A., Blickle, A., Paolini, S., Ohlinger, L., \& Forrey, R. C. 2012, Phys. Rev. A, 85, 042710

Firsov O.B., 1951, Zh. Eksp. Teor. Fiz., 21, 1001

Janev, R. K., Langer, W. D., \& Evans, K. 1987, Springer Series on Atoms and Plasmas, Berlin: Springer, 1987

Mashonkina, L. 2009, Physica Scripta Volume T, 134, 014004

Mashonkina, L. 2010, EAS Publications Series, 43, 79

Mihajlov, A. A. \& Janev, R. K. 1981, J. Phys. B: At. Mol. Opt. Phys., 14, 1639

Mihajlov, A. A., Ignjatović, L. M., Srećković, V. A., \& Dimitrijević, M. S. 2011a, ApJS, 193, 2

Mihajlov, A. A., Ignjatović, L. M., Srećković, V. A., \& Dimitrijević, M. S. 2011b, Baltic Astronomy, 20, 566

Mihajlov, A. A., Srećković, V. A., Ignjatović, L. M., \& Klyucharev, A.N. 2012, Journal of Cluster Science, 23 (1), 47

Mihajlov, A. A., Ignjatović, L. M., Sakan, N. M., \& Dimitrijević, M. S. 2007a, A\&A, 469, 749

Mihajlov, A. A., Ignjatovic, L. M., Djuric, Z., \& Ljepojevic, N. N. 2004, Journal of Physics B Atomic Molecular Physics, 37, 4493

Mihajlov, A. A., Jevremović, D., Hauschildt, P., et al. 2007b, A\&A, 471, 671

Mihajlov, A. A., Jevremović, D., Hauschildt, P., et al. 2003, A\&A, 403, 787

Ryabtsev, I. I., Tretyakov, D. B., Beterov, I. I., et al. 2005, Journal of Physics B Atomic Molecular Physics, 38, 17

Sobelman, I. I. 1979, Springer Series in Chemical Physics, Berlin: Springer, 1979,

Srećković, V. A., Mihajlov, A. A., Ignjatović, L. M., \&

Dimitrijević, M. S. 2013, A\&A, 552, A33

Urbain, X., Cornet, A., Brouillard, F., \& Giusti-Suzor, A. 1991, Phys.Rev.Lett., 66, 1685

\section{APPENDIX}

\section{APPENDIX MATERIAL}

The characteristic length $R_{u p ; m i x}$ is defined here as the upper limit of the domain $R$ where at given $E$ and $\rho$ we can consider that the inner electron is in the subsystem $\mathrm{H}^{+}+\mathrm{H}(1 s)$ and that is sufficiently delocalized, so that this subsystem could be treated as a quasi-molecular complex. As a qualitative characteristics of the mentioned delocalization, one takes here the probability of resonant charge exchange $P_{c . e x c}(R ; E ; \rho)$ in the subsystem $\mathrm{H}^{+}+\mathrm{H}(1 s)$ as a function of $R$ at given $\rho$ and $E$. As the basis for this, is taken the theory of the process: $\mathrm{H}^{+}+\mathrm{H}(1 s) \rightarrow \mathrm{H}(1 s)+\mathrm{H}^{+}$, developed in Firsov (1951) and Bates \& Boyd (1962). From this theory follows that

$$
P_{\text {c.exc }}(R ; E ; \rho)=\sin ^{2}(\varphi(R ; E ; \rho)),
$$

where the phase $\varphi(R ; E ; \rho)$ is given by the relation

$$
\varphi(R ; E ; \rho)=\frac{1}{2} \int_{R}^{\infty} \frac{U_{12}\left(R^{\prime}\right)}{v_{\text {rad }}\left(R^{\prime}, \rho, E\right)} d R^{\prime},
$$

which can be used in the considered case since $P_{\text {c.exc }}(R ; E ; \rho)$ becomes noticeably different from zero only deeply inside the orbit of Rydberg electron at given $n$. On the basis of data from Firsov (1951) and Bates \& Boyd (1962) can be considered that in the case when $P_{c . e x c}(R ; E ; \rho)$ reaches the value of $1 / 2 \pi$ the corresponding $R$ may be considered as the upper limit of the charge exchange zone at given $\rho$ and $E$. And consequently, as the upper limit of domain with a sufficient degree of delocalization of electron in the subsystem $\mathrm{H}^{+}+\mathrm{H}(1 s)$. Consequently, the parameter $R_{u p ; m i x}$ is determined here as the root of equation

$$
\sin ^{2}(\varphi(R ; E ; \rho))=\frac{1}{2 \pi}
$$

where $\varphi(R ; E ; \rho)$ is given by Eq. A2 under condition that this root is in the domain of monotonical increase of the left side of Eq. A3 .

The behavior of phase $\varphi(R ; E ; \rho)$ is illustrated by Tab. 3. where its values for $E=E_{n ; i}, \rho=0$ and $R=R_{n ; n+1}$ within the range $3 \leq n \leq 15$ are shown. Of course, these data should be treated as the qualitative ones since Eqs. A1 and (A2) have strict sense in the case $E \gg U_{12}(R)$ while in our case when this condition is fulfilled only for $n>7$. 
Table 1

Calculated values of the coefficient $K_{1 ; n}(T)\left(\mathrm{cm}^{3} \mathrm{~s}^{-1}\right)$ as a function of $n$ and $T$.

\begin{tabular}{|c|c|c|c|c|c|c|c|c|c|c|c|c|c|}
\hline & & & & & & $\mathrm{n}$ & & & & & & & \\
\hline$T$ & 3 & 4 & 5 & 6 & 7 & 8 & 9 & 10 & 11 & 12 & 13 & 14 & 15 \\
\hline 4000 & $7.17 \mathrm{E}-12$ & $1.54 \mathrm{E}-10$ & $3.58 \mathrm{E}-10$ & $4.28 \mathrm{E}-10$ & $3.98 \mathrm{E}-10$ & $3.30 \mathrm{E}-10$ & $2.61 \mathrm{E}-10$ & $2.06 \mathrm{E}-10$ & $1.63 \mathrm{E}-10$ & $1.28 \mathrm{E}-10$ & $1.02 \mathrm{E}-10$ & $8.14 \mathrm{E}-11$ & $6.62 \mathrm{E}-11$ \\
\hline 4250 & $9.01 \mathrm{E}-12$ & $1.63 \mathrm{E}-10$ & $3.88 \mathrm{E}-10$ & $4.52 \mathrm{E}-10$ & $4.15 \mathrm{E}-10$ & $3.42 \mathrm{E}-10$ & $2.69 \mathrm{E}-10$ & $2.11 \mathrm{E}-10$ & $1.66 \mathrm{E}-10$ & $1.31 \mathrm{E}-10$ & $1.04 \mathrm{E}-10$ & $8.28 \mathrm{E}-11$ & $6.72 \mathrm{E}-11$ \\
\hline 4500 & $1 \mathrm{E}-11$ & $1.72 \mathrm{E}-10$ & $4.16 \mathrm{E}-10$ & $4.76 \mathrm{E}-10$ & $4.32 \mathrm{E}-10$ & $3.53 \mathrm{E}-10$ & $2.76 \mathrm{E}-10$ & $2.16 \mathrm{E}-10$ & $1.70 \mathrm{E}-10$ & $1.33 \mathrm{E}-10$ & $1.06 \mathrm{E}-10$ & $8.40 \mathrm{E}-11$ & $81 \mathrm{E}-11$ \\
\hline 4750 & $1.33 \mathrm{E}-11$ & $1.83 \mathrm{E}-10$ & $4.43 \mathrm{E}-10$ & $4.98 \mathrm{E}-10$ & $4.48 \mathrm{E}-10$ & $3.63 \mathrm{E}-10$ & $2.83 \mathrm{E}-10$ & $2.20 \mathrm{E}-10$ & $1.73 \mathrm{E}-10$ & $1.35 \mathrm{E}-10$ & $1.07 \mathrm{E}-10$ & $.51 \mathrm{E}-11$ & $6.89 \mathrm{E}-11$ \\
\hline 5000 & $1.53 \mathrm{E}-11$ & $1.96 \mathrm{E}-10$ & $4.71 \mathrm{E}-10$ & $5.20 \mathrm{E}-10$ & $4.63 \mathrm{E}-10$ & $72 \mathrm{E}-10$ & $2.89 \mathrm{E}-10$ & $2.24 \mathrm{E}-10$ & $1.76 \mathrm{E}-10$ & $38 \mathrm{E}-10$ & $1.09 \mathrm{E}-10$ & $62 \mathrm{E}-11$ & $6.97 \mathrm{E}-11$ \\
\hline 5250 & $1.73 \mathrm{E}-11$ & $2.12 \mathrm{E}-10$ & $4.98 \mathrm{E}-10$ & $5.42 \mathrm{E}-10$ & $4.77 \mathrm{E}-10$ & $81 \mathrm{E}-10$ & $2.95 \mathrm{E}-10$ & $2.28 \mathrm{E}-10$ & $1.78 \mathrm{E}-10$ & $1.40 \mathrm{E}-10$ & $1.10 \mathrm{E}-10$ & $8.73 \mathrm{E}-11$ & $7.05 \mathrm{E}-11$ \\
\hline 5500 & $1.96 \mathrm{E}-11$ & $2.31 \mathrm{E}-10$ & $5.26 \mathrm{E}-10$ & $5.63 \mathrm{E}-10$ & $4.90 \mathrm{E}-10$ & $3.89 \mathrm{E}-10$ & $3.01 \mathrm{E}-10$ & $2.31 \mathrm{E}-10$ & $1.80 \mathrm{E}-10$ & $1.41 \mathrm{E}-10$ & $1.11 \mathrm{E}-10$ & $8.84 \mathrm{E}-11$ & $7.12 \mathrm{E}-11$ \\
\hline 5750 & $2.30 \mathrm{E}-11$ & 2.51 & $5.53 \mathrm{E}-10$ & $5.83 \mathrm{E}$ & & $6 \mathrm{E}$ & & & $1.82 \mathrm{E}-10$ & $1.43 \mathrm{~F}$ & & $8.94 \mathrm{E}-11$ & $7.19 \mathrm{E}-11$ \\
\hline 6000 & $2.81 \mathrm{E}-11$ & $2.71 \mathrm{E}-10$ & $5.79 \mathrm{E}-10$ & $6.03 \mathrm{E}-10$ & $5.15 \mathrm{E}-10$ & $4.04 \mathrm{E}-10$ & $3.11 \mathrm{E}-10$ & $2.38 \mathrm{E}-10$ & $1.84 \mathrm{E}-10$ & $1.44 \mathrm{E}-10$ & $1.14 \mathrm{E}-10$ & $9.03 \mathrm{E}-11$ & $7.25 \mathrm{E}-11$ \\
\hline 6250 & & 10 & & & & & & & & & & & E-11 \\
\hline 6500 & $4.37 \mathrm{E}-11$ & $3.11 \mathrm{E}-10$ & $6.26 \mathrm{E}-10$ & $6.39 \mathrm{E}-10$ & $5.37 \mathrm{E}-10$ & $4.17 \mathrm{E}-10$ & $3.20 \mathrm{E}-10$ & $2.44 \mathrm{E}-10$ & $1.88 \mathrm{E}-10$ & $1.47 \mathrm{E}-10$ & $1.16 \mathrm{E}-10$ & $9.19 \mathrm{E}-11$ & $7.36 \mathrm{E}-11$ \\
\hline 7000 & & 3.50 & & & & & & & & & & 11 & $6 \mathrm{E}-11$ \\
\hline 7500 & $7.08 \mathrm{E}-11$ & $3.90 \mathrm{E}-10$ & $7.13 \mathrm{E}-10$ & $7.03 \mathrm{E}-10$ & $5.80 \mathrm{E}-10$ & $4.43 \mathrm{E}-10$ & $3.36 \mathrm{E}-10$ & $2.55 \mathrm{E}-10$ & $1.95 \mathrm{E}-10$ & $1.51 \mathrm{E}-10$ & $1.20 \mathrm{E}-10$ & $9.46 \mathrm{E}-11$ & $7.57 \mathrm{E}-11$ \\
\hline & & & & & & & & 2. & & & & E-11 & $7.68 \mathrm{E}-11$ \\
\hline 8500 & $8.91 \mathrm{E}-11$ & $4.71 \mathrm{E}-10$ & $7.93 \mathrm{E}-10$ & $7.57 \mathrm{E}-10$ & $6.14 \mathrm{E}-10$ & $4.65 \mathrm{E}-10$ & $3.51 \mathrm{E}-10$ & $2.64 \mathrm{E}-10$ & $2.01 \mathrm{E}-10$ & $1.56 \mathrm{E}-10$ & $1.22 \mathrm{E}-10$ & $9.66 \mathrm{E}-11$ & $7.75 \mathrm{E}-11$ \\
\hline & $9.91 \mathrm{E}-11$ & $5.13 \mathrm{E}-10$ & $8.27 \mathrm{E}-10$ & $7.82 \mathrm{E}-10$ & E-10 & 4.7 & E-10 & $2.68 \mathrm{E}-10$ & E-10 & E-10 & $1.23 \mathrm{E}-10$ & $4 \mathrm{E}-11$ & $7.81 \mathrm{E}-11$ \\
\hline 9500 & $1.06 \mathrm{E}-10$ & $5.56 \mathrm{E}-10$ & $8.57 \mathrm{E}-10$ & $8.06 \mathrm{E}-10$ & $6.40 \mathrm{E}-10$ & $4.82 \mathrm{E}-10$ & $3.61 \mathrm{E}-10$ & $2.72 \mathrm{E}-10$ & $2.06 \mathrm{E}-10$ & $1.59 \mathrm{E}-10$ & $1.25 \mathrm{E}-10$ & $9.82 \mathrm{E}-11$ & $7.86 \mathrm{E}-11$ \\
\hline 10000 & $1.07 \mathrm{E}-10$ & $6.03 \mathrm{E}-10$ & $8.82 \mathrm{E}-10$ & $8.30 \mathrm{E}-10$ & $6.55 \mathrm{E}-10$ & $4.90 \mathrm{E}-10$ & $3.66 \mathrm{E}-10$ & $2.75 \mathrm{E}-10$ & $2.08 \mathrm{E}-10$ & $1.61 \mathrm{E}-10$ & $1.26 \mathrm{E}-10$ & $9.91 \mathrm{E}-11$ & 7.93E-11 \\
\hline
\end{tabular}


Table 2

Calculated values of the branch coefficient $X_{1 b ; n}$ as a function of $n$ and $T$.

\begin{tabular}{|c|c|c|c|c|c|c|c|c|c|c|c|c|c|}
\hline \multicolumn{14}{|c|}{$\mathrm{n}$} \\
\hline$T$ & 3 & 4 & 5 & 6 & 8 & 8 & 9 & 10 & 11 & 12 & 13 & 14 & 15 \\
\hline 4000 & 0.684 & 0.608 & 0.458 & 0.365 & 0.306 & 0.243 & 0.218 & 0.208 & 0.201 & 0.186 & 0.169 & 0.154 & 0.137 \\
\hline 4250 & 0.607 & 0.563 & 0.437 & 0.346 & 0.284 & 0.232 & 0.211 & 0.202 & 0.195 & 0.178 & 0.160 & 0.143 & 0.129 \\
\hline 4500 & 0.543 & 0.519 & 0.421 & 0.329 & 0.265 & 0.222 & 0.206 & 0.198 & 0.189 & 0.170 & 0.152 & 0.132 & 0.122 \\
\hline 4750 & 0.497 & 0.475 & 0.407 & 0.314 & 0.248 & 0.213 & 0.201 & 0.194 & 0.184 & 0.162 & 0.144 & 0.122 & 0.114 \\
\hline 5000 & 0.467 & 0.431 & 0.395 & 0.301 & 0.232 & 0.205 & 0.197 & 0.190 & 0.180 & 0.155 & 0.137 & 0.112 & 0.108 \\
\hline 5250 & 0.473 & 0.427 & 0.370 & 0.284 & 0.223 & 0.201 & 0.193 & 0.185 & 0.172 & 0.146 & 0.130 & 0.109 & 0.104 \\
\hline 5500 & 0.467 & 0.419 & 0.347 & 0.269 & 0.215 & 0.197 & 0.189 & 0.181 & 0.164 & 0.137 & 0.123 & 0.107 & 0.101 \\
\hline 5750 & 0.442 & 0.411 & 0.328 & 0.254 & 0.208 & 0.193 & 0.186 & 0.177 & 0.157 & 0.128 & 0.116 & 0.104 & 0.098 \\
\hline 6000 & 0.397 & 0.403 & 0.310 & 0.242 & 0.201 & 0.190 & 0.183 & 0.173 & 0.150 & 0.119 & 0.110 & 0.101 & 0.095 \\
\hline 6250 & 0.349 & 0.380 & 0.294 & 0.229 & 0.197 & 0.187 & 0.179 & 0.166 & 0.142 & 0.116 & 0.107 & 0.099 & 0.092 \\
\hline 6500 & 0.308 & 0.360 & 0.279 & 0.218 & 0.194 & 0.184 & 0.175 & 0.159 & 0.134 & 0.113 & 0.104 & 0.096 & 0.089 \\
\hline 7000 & 0.263 & 0.327 & 0.254 & 0.198 & 0.187 & 0.178 & 0.169 & 0.146 & 0.118 & 0.106 & 0.098 & 0.092 & 0.083 \\
\hline 7500 & 0.223 & 0.292 & 0.234 & 0.190 & 0.180 & 0.172 & 0.157 & 0.133 & 0.112 & 0.101 & 0.093 & 0.086 & 0.078 \\
\hline 8000 & 0.199 & 0.263 & 0.216 & 0.183 & 0.173 & 0.167 & 0.146 & 0.120 & 0.105 & 0.096 & 0.087 & 0.081 & 0.073 \\
\hline 8500 & 0.198 & 0.243 & 0.194 & 0.178 & 0.169 & 0.161 & 0.134 & 0.112 & 0.100 & 0.092 & 0.083 & 0.075 & 0.070 \\
\hline 9000 & 0.198 & 0.225 & 0.175 & 0.173 & 0.165 & 0.156 & 0.123 & 0.105 & 0.095 & 0.087 & 0.080 & 0.069 & 0.067 \\
\hline 9500 & 0.201 & 0.210 & 0.164 & 0.167 & 0.161 & 0.142 & 0.116 & 0.099 & 0.091 & 0.084 & 0.075 & 0.067 & 0.065 \\
\hline 10000 & 0.218 & 0.196 & 0.155 & 0.161 & 0.156 & 0.129 & 0.109 & 0.095 & 0.087 & 0.081 & 0.071 & 0.066 & 0.063 \\
\hline
\end{tabular}

Table 3

Calculated values of the parameters which characterize pre-ionization zone. Phase $\varphi\left(R_{n, n+1}, E_{n ; i} ; \rho=0\right)$ is given by the relation Eq.

(A2).

\begin{tabular}{cccccc}
\hline$n$ & $R_{n i}$ & $E_{n ; i}=U 2(R n ; i)$ & $R_{n, n+1}$ & $\varphi\left(R_{n, n+1}, E_{n ; i} ; \rho=0\right)$ & $P_{c . e x c}\left(\varphi\left(R_{n, n+1}, E_{n ; i} ; \rho=0\right)\right)$ \\
\hline 3 & 4.79 & 0.02738 & 5.839 & 1.840 & 0.92929 \\
4 & 5.52 & 0.01431 & 6.777 & 1.143 & 0.82824 \\
5 & 6.08 & 0.00871 & 7.497 & 0.782 & 0.49618 \\
6 & 6.52 & 0.00581 & 8.087 & 0.567 & 0.28891 \\
7 & 6.89 & 0.00413 & 8.580 & 0.433 & 0.17619 \\
8 & 7.21 & 0.00306 & 9.010 & 0.341 & 0.07544 \\
9 & 7.49 & 0.00234 & 9.380 & 0.278 & 0.05167 \\
10 & 7.73 & 0.00183 & 9.725 & 0.229 & 0.03667 \\
11 & 7.95 & 0.00146 & 10.035 & 0.193 & 0.02691 \\
12 & 8.16 & 0.00119 & 10.317 & 0.165 & 0.02108 \\
13 & 8.34 & 0.00098 & 10.551 & 0.146 & 0.01489 \\
14 & 8.51 & 0.00081 & 10.839 & 0.122 & 0.01297 \\
15 & 8.66 & 0.00068 & 11.002 & 0.114 & \\
\hline
\end{tabular}

